\title{
Ultra-Small Silver Nanoparticles Immobilized in Mesoporous SBA-15. Microwave-Assisted Synthesis and Catalytic Activity in the 4-Nitrophenol Reduction
}

\author{
Roberta Manno $^{\mathrm{a}, \mathrm{c}}$, Victor Sebastian ${ }^{\mathrm{a}, \mathrm{b}, \mathrm{c}, *}$, Silvia Irusta ${ }^{\mathrm{a}, \mathrm{b}, \mathrm{c}}$, Reyes Mallada ${ }^{\mathrm{a}, \mathrm{b}, \mathrm{c}, *}$, \\ Jesús Santamaria ${ }^{\text {a,b,c }}$ \\ ${ }^{a}$ Nanoscience Institute of Aragon and Chemical and Environmental Engineering Department, University of Zaragoza, 50018, Zaragoza, Spain \\ ${ }^{\mathrm{b}}$ Networking Research Center CIBER-BBN, 28029, Madrid, Spain \\ ${ }^{\mathrm{c}}$ Aragón Materials Science Institute, ICMA, CSIC - University of Zaragoza, Pedro Cerbuna 12, 50009, Zaragoza, Spain
}

\section{A R T I C L E I N F O}

\section{Keywords:}

Microwave

Silver Nanoparticles

Mesoporous SBA-15

Catalyst

4-Nitrophenol

\begin{abstract}
A B S T R A C T
Despite the continuous developments in the synthesis of noble metal nanoparticles, the uniformity of particle size distribution still represents a critical aspect. A fast and homogeneous nucleation is a key requirement to achieve a monodisperse particle size distribution and in this scenario, the application of alternative energy sources may constitute a winning strategy for the development of highly active nanocatalysts with unique properties. Here we present several approaches to control the synthesis of Ag nanoparticles stabilized by an anionic template, and the results evidence the advantages of adopting unconventional heating techniques such as microwave heating. The fast and selective electromagnetic heating strongly reduced the nucleation and growth times, impacting on the homogeneity of the resulting particle size distribution. In this work, we have carried out the microwave-assisted synthesis of Ag nanoparticles and the resulting nanoparticles were compared to those synthesized under conventional heating using an oil bath, showing that the differences in temperature profile and heating rates between the two synthesis pathways had a clear effect on the size distribution of the resulting nanoparticles as well as on their stability under long term storage. Finally, the synthesized ultra-small Ag nanoparticles were deposited on a mesoporous substrate, reducing undesired Ostwald ripening and facilitating their reusability. This nanocatalyst was adopted for the abatement of 4-nitrophenol, a well-known carcinogenic pollutant with adverse effects on human beings and aquatic life. The catalytic results confirm the high activity of the catalyst thanks to the high dispersion achieved afforded by ultra-small Ag nanoparticles and the accessibility provided by the wide SBA-15 mesoporous channels.
\end{abstract}

\section{Introduction}

A crucial problem of the synthesis of nanostructured catalytic materials is still the poor control of their size distribution. The generation of secondary products or particles with different diameters may strongly affect not only their long-time stability but also their catalytic properties [1,2]. In 1935 Becker and Döring introduced a thermodynamic model, known as Classical Nucleation Theory (CNT), which expresses the Gibbs free energy of a spherical particle as function of its radius. If the radius is higher than a critical value $r_{c}$, which depends on the ratio of the surface energy per unit area and the Gibbs free energy per unit volume, the energy of the system exceeds the activation energy and enhances the particle growth $[3,4]$. The nucleation rate can be described by the Arrhenius equation as a function of the Gibbs free energy and the temperature of the system. As reported by Graham et al. [5], longer nucleation time or higher temperature may promote the simultaneity of nucleation and coalescence mechanisms, reducing the homogeneity of the particle size distribution. This is especially important when monodisperse ultra-small nanoparticles (NPs) with a diameter smaller than $2 \mathrm{~nm}$ are the objective. These are desirable because their high surface to volume ratio, together with unusual quantum size effects are the hallmarks of an innovative category of highly active nanocatalysts [6-8].

The way the energy needed for particle nucleation is supplied is key in the growth process and a wide range of alternative energy sources have been proposed for the controlled production of ultra-small $\mathrm{Ag}$ -

\footnotetext{
* Corresponding authors.

E-mail addresses: victorse@unizar.es (V. Sebastian), rmallada@unizar.es (R. Mallada).
} 
a.

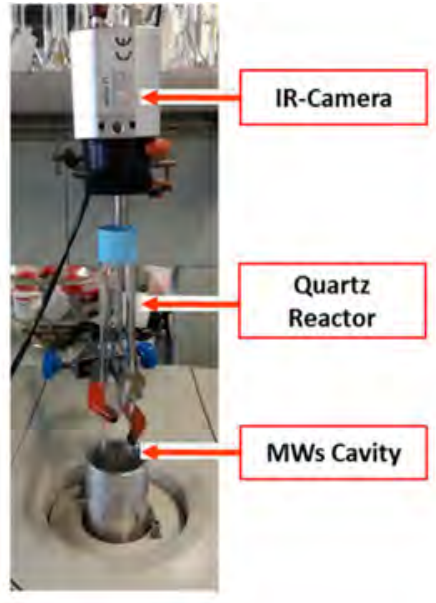

b.

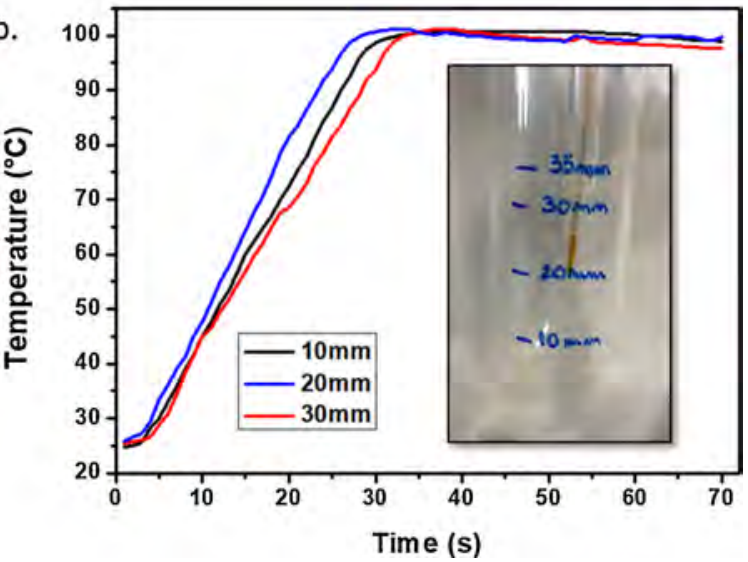

Fig. 1. (a) Quartz batch reactor (I.D. $20.8 \mathrm{~mm}$, O.D. $24.0 \mathrm{~mm}$ ) with the evidence of the IR camera for the analysis of radial temperature profile and (b) Evolution of temperature in a representative synthesis of Ag NPs (synthesis time of $70 \mathrm{~s}$, input power of $200 \mathrm{~W}$ ) as a function of MW irradiation time at different positions of the optical fiber 10,20 and $30 \mathrm{~mm}$ from the bottom part, (see inset optical image) ensuring total immersion of the fiber since the total height of the liquid corresponds to $35 \mathrm{~mm}$.
NPs. Generally, the aim is to achieve a rapid nucleation step, and sources such as $\gamma$-irradiation [9-11] microwave heating [12,13], UVirradiation [14] or sonochemical method [15] have been studied. From their comparison, microwaves (MW) seem most advantageous, giving a noticeable reduction of the synthesis time down to $70 \mathrm{~s}$ and a strong improvement of the size distribution [12]. Despite these encouraging results, considerable room for improvement exists, especially concerning the analysis of the heating patterns and the possibility of temperature gradients in the system. Furthermore, the deposition of small nanoparticles on a suitable substrate needs further study as it may both stabilize the NPs and facilitate their application in heterogeneous catalytic reactions, allowing its reusability [16-18].

In this work, we report an investigation of a rapid MW-assisted synthesis of ultra-small Ag-NPs. After studying the effect of irradiation time, we compared the nanoparticles obtained under MW heating with those under conventional heating (immersion in an oil bath), focusing the discussion on the differences of the temperature patterns in both systems. We have also studied the stability of the NPs formed upon long term storage i.e. 18 months. Finally, the homogeneous ultra-small AgNPs were deposited on a porous substrate and used in the catalytic reduction of 4-Nitrophenol (4-NP) in water. This is often used as a test reaction since 4-NP is widely used in a number of manufacturing processes, including drugs, pesticides and leather treatment, resulting in environmental and health hazards [19-21].

\section{Materials and Methods}

\subsection{Synthesis of amine grafted $S B A-15$}

A traditional sol-gel procedure was adopted for the synthesis of mesoporous SBA-15 nanorods, previously reported by Uson et al. [22]. Summarily, $1.2 \mathrm{~g}$ of Poly(ethylene Glycol) - block - poly(propylene glycol) - block - poly(ethylene glycol) (Pluronic P-123, Sigma-Aldrich) and $14 \mathrm{mg}$ of ammonium fluoride $\mathrm{NH}_{4} \mathrm{~F}$ (Sigma Aldrich, $\geq 98 \%$ ) were dissolved into $40 \mathrm{~mL}$ of $\mathrm{HCl} 1.75 \mathrm{M}$ (AnalaR NORMAPUR 37\%), maintained at $20^{\circ} \mathrm{C}$ until complete dissolution of the copolymer. Then, a mixture of heptane (Sigma-Aldrich, 99\%) $(8.5 \mathrm{~mL})$ and TEOS (Fluka, $98 \%)(2.75 \mathrm{~mL})$ was added, keeping the reaction under vigorous stirring for $4 \mathrm{~min}$. After $10 \mathrm{~min}$ of static conditions, the solution was transferred to a PTFE-lined autoclave for hydrothermal treatment at $100{ }^{\circ} \mathrm{C}$ for $24 \mathrm{~h}$. The washed and filtered product was calcined in a muffle furnace at $550{ }^{\circ} \mathrm{C}$ for $5 \mathrm{~h}$ with a heating rate of $1{ }^{\circ} \mathrm{C} / \mathrm{min}$ to remove the template. For subsequent amine-grafting, a mass of $1 \mathrm{~g}$ of calcined SBA-15 nanorods was dispersed into $20 \mathrm{ml}$ of toluene (Fisher Chemical, General Purpose Grade). The system was purged with argon for $15 \mathrm{~min}$ and mechanically stirred at $800 \mathrm{rpm}$. Then, $400 \mu \mathrm{L}$ of (3aminopropyl)triethoxysilane (Sigma-Aldrich, $\geq 98 \%$ ) were added and the system was maintained at $110^{\circ} \mathrm{C}$ for $1 \mathrm{~h}$. Finally, the product was filtered, washed with a mixture of dichlorometane/ diethyl ether (1:1 in volume) and dried at $80^{\circ} \mathrm{C}$ overnight.

\subsection{Synthesis of Ag nanoparticles}

Microwave-assisted synthesis of Ag NPs was conducted in CEM Microwave Discover ${ }^{\circledR}$ Cavity. The quartz reactor (ID $20.8 \mathrm{~mm}$, OD $24.0 \mathrm{~mm}$ ) was precisely fixed in the centre of the open vessel cavity. For the synthesis of Ag NPs, $85 \mathrm{mg}$ of silver nitrate (Aldrich 99.9999\%) were dissolved under mechanical stirring at room temperature in $10 \mathrm{~mL}$ of deionized water $(50 \mathrm{mM})$ and covered with aluminium foil. Then, $500 \mu \mathrm{L}$ of poly (acrylic acid, sodium salt) solution (Na-PAA Sigma Aldrich MW 1200, 45 wt \% in water) was added and high stirring was applied to enssure a complete mixing of the reagents. The mixture was irradiated by microwaves, with and input power of $200 \mathrm{~W}$, for three different time-lapses (17 s, $35 \mathrm{~s}$ and $70 \mathrm{~s}$ ) under high stirring condition (magnetic stirrer bar of $\emptyset 4 \mathrm{~mm} \times 10 \mathrm{~mm}$ ). Na-PAA played the dual role of reducing and stabilizing agent. For conventional heating $(\mathrm{CH})$ we adopted a traditional oil bath previously set at the desired working temperature, i.e. $74.6{ }^{\circ} \mathrm{C}$. Accurate monitoring of reaction temperature was performed both by a thermographic camera (Optris PI400038T900) that recorded the radial temperature profile on the top surface and by an optical fiber at three different axial points $(10,20$ and $30 \mathrm{~mm}$ ) of the $35 \mathrm{~mm}$ of the height occupied by the liquid volume as reported in Fig. $1 \mathrm{a}$ and $\mathrm{b}$ respectively. The observed temperature gradient is due to a non-uniform distribution of the electromagnetic field, already observed by other authors with the same cavity [23].

To stabilize the synthesized NPs and to allow their application for heterogeneous catalytic reactions, $100 \mathrm{mg}$ of amino-functionalized SBA-15 nanorods were impregnated by $400 \mu \mathrm{l}$ of the as-made PAA-Ag colloid (1 h x1500 rpm). Afterward, the catalyst was centrifuged with distilled water (12000 rpm x20 min), eliminating non-grafted NPs, and dried in an oven at $50^{\circ} \mathrm{C}$ overnight.

\subsection{Characterization techniques}

The effects of PAA-Ag NPs grafting on specific surface area and pore volume distribution of amine-functionalized SBA-15 were evaluated by nitrogen adsorption analysis at $77 \mathrm{~K}$ in a Micrometrics ASAP 2020. The samples were out-gassed at $26.7 \mathrm{~Pa}$ and $383 \mathrm{~K}$ for $5 \mathrm{~h}$ before the measurement. The analysis of the adsorption branch by Brunauer-JoynerHalenda (BJH) method was adopted for the calculation of mean pore diameter (MPD) and pore size distribution. Brunauer-Emmett-Teller (BET) method was applied for the determination of specific surface area from the adsorption isotherm in the range of relative pressure 0.060.19 . 
Transmission Electron Microscopy (TEM) analysis was conducted to characterize the synthesized $\mathrm{Ag}$ nanoparticles and their dispersion on Ag-NPs/SBA-15 nanorods. A $10 \mu \mathrm{l}$ drop of colloidal nanoparticles was deposited onto a Formvar TEM copper grid covered by a continuous carbon film. After complete evaporation, the sample was analysed in a FEI TECNAI T20 microscope with an operating voltage of $200 \mathrm{keV}$. A similar procedure was adopted for the characterization of ultra-small Ag-NPs before and after grafting on silica substrate, dropping $10 \mu \mathrm{L}$ suspension of the PAA-Ag NPs onto a holey carbon TEM grid. In this case, a high-angle annular dark-field scanning transmission electron microscope FEI Titan $^{\mathrm{TM}}(80-300 \mathrm{kV})$ was adopted and elemental analysis was carried out with an EDS detector that allows performing EDS experiments in the scanning mode. These two microscopes belong to the Laboratory of Advanced Microscopies (LMA) at the Institute of Nanoscience of Aragon (INA), University of Zaragoza.

The optical properties of PAA-Ag particles were evaluated by UV-Vis Spectroscopy (Agilent 8453 UV-Visible Spectrophotometer). $500 \mu \mathrm{L}$ of the sample was diluted into $2.5 \mathrm{~mL}$ of deionized water. The measurement range adopted was from 1000 to $200 \mathrm{~nm}$ with a scan speed of $400 \mathrm{~nm} / \mathrm{min}$.

The quantification of Ag metal loading in the mesoporous channels was determined by Microwave Plasma Atomic Emission Spectroscopy (MP-AES) (Agilent $4100 \mathrm{MP}-\mathrm{AES}$ ). Firstly, $30 \mathrm{mg}$ of the catalyst were microwave digested $\left(200^{\circ} \mathrm{C}\right.$ for $20 \mathrm{~min}$ in Milestone Ethos Plus microwave cavity) in $5 \mathrm{ml}$ of nitric acid $\left(\mathrm{HNO}_{3}\right)$ and hydrochloric acid $(\mathrm{HCl})$ in a volume ratio of $1: 3$. Then, the digested sample was diluted with Milli-Q water obtaining a final volume of $30 \mathrm{ml}$. To discard any fragmented particle, the sample was centrifuged (12000 rpm x20 min) and the supernatant was filtered by hydrophilic syringe filters of $0.2 \mu \mathrm{m}$.

The presence of $\mathrm{Ag}_{\mathrm{m}+\mathrm{n}}{ }^{\mathrm{m}+}$ species was verified by X-ray photoelectron spectroscopy (XPS), performed with an Axis Supra spectrometer (Kratos Tech). The samples were mounted on a sample rod placed in the pre-treatment chamber of the spectrometer and then evacuated at room temperature. The spectra were excited by a monochromatized $\mathrm{AlK}_{\alpha}$ source at $1486.6 \mathrm{eV}$ and subsequently run at $12 \mathrm{kV}$ and $10 \mathrm{~mA}$. Survey spectrum was measured at $160 \mathrm{eV}$ pass energy and for the individual peak regions, spectra were recorded with a pass energy of $20 \mathrm{eV}$. CasaXPS software was adopted to analyse the peaks adopting a weighted sum of Lorentzian and Gaussian component curves after Shirley background subtraction. The binding energies were referenced to the internal $\mathrm{C}_{1 \mathrm{~s}}$ standard at $284.9 \mathrm{eV}$.

\subsection{Catalytic Activity}

Reduction of 4-Nitrophenol by an excess of $\mathrm{NaBH}_{4}$ in water was selected to test the catalytic activity of PAA-Ag nanoparticles supported on SBA-15. In a typical reaction, $1.44 \mathrm{mmol}_{\text {of }} \mathrm{NaBH}_{4}$ was added into $30 \mathrm{ml}$ of a fresh solution of 4-Nitrophenol $(0.125 \mathrm{mM})$. After collecting the UV-Vis spectrum as a reference of time zero, $1 \mathrm{mg} / \mathrm{l}$ of the catalyst was added keeping the reaction in stirring condition, covered with Aluminium foil. The absorbance peak was analysed every $40 \mathrm{~s}$ in a continuous UV-Vis Spectrophotometer (Agilent 8453). For reusability test, the solution was centrifuged after each cycle, observing the deposition of the nanocatalyst as a pellet. The supernatant solution was eliminated, the residue was dried and then reused for a different cycle.

\section{Results and discussion}

\subsection{Synthesis of Ultra-Small Ag nanoparticles}

Firstly, we performed a detailed analysis of the microwave-assisted synthesis of PAA-Ag NPs, evaluating the effects of three different irradiation times ( $17 \mathrm{~s}, 35 \mathrm{~s}$ and $70 \mathrm{~s}$ ). A poor control of particle size distribution was observed for nanoparticles synthesized at $70 \mathrm{~s}$ and $35 \mathrm{~s}$, as reported in Fig. 2 a) and Fig. 2 c), respectively. While more than $85 \%$ of nanoparticles presented a diameter smaller than $3 \mathrm{~nm}$, a significant proportion of particles with a size around $10 \mathrm{~nm}$ were observed in both cases. Instead, a uniform size distribution of $(1.6 \pm 0.7) \mathrm{nm}$ was observed for NPs produced with an irradiation time of $17 \mathrm{~s}$, Fig. 2 e. In accordance with LaMer model, the fast microwave heating $\left(2.8^{\circ} \mathrm{C} / \mathrm{s}\right.$ as reported in Fig. 1-b and Figure S1) enhanced uniform nucleation of small particles. However, the subsequent, or even simultaneous, Ostwald ripening and diffusion mechanisms are responsible of the growth of the initial nuclei which may be strongly accelerated by higher temperatures [3]. For this reason, we fixed reagents concentrations, reactor configuration, heating rate and the procedure for the characterization of the samples, focusing on final temperature and synthesis time. At a heating time of $17 \mathrm{~s}$, the maximum temperature reached was of $(74.6 \pm 6.6){ }^{\circ} \mathrm{C}, 23 \%$ lower than the maximum temperature used for $35 \mathrm{~s}$ and $70 \mathrm{~s}$ (Fig. 1 and Figure S1). According to studies using in situ SAXS at a millisecond time resolution, colloidal silver nanoparticles grow via the cluster-aggregative nucleation pathway [24]. This pathway implies that silver ions are reduced to produce $\mathrm{Ag}_{13}$ clusters that subsequently form small silver nanoparticles by agglomeration of $\mathrm{Ag}_{13}$ clusters. Temperature significantly influences the nucleation event, modifying the maximum concentration supersaturation and the diffusion mobility of $\mathrm{Ag}_{13}$ clusters. Considering this fact, it was observed that increasing the temperature from $60^{\circ} \mathrm{C}$ to $92^{\circ} \mathrm{C}$ resulted in the presence of successive nucleation that introduced non-uniformity in the growth kinetics of Ag NPs due to unavoidable random coagulations/ aggregations events [25]. In fact, the aggregation kinetics increase with temperature [26]. In our analysis, no relevant aggregation was reported up to $75^{\circ} \mathrm{C}$, where a unique population was observed. Instead, a consistent cluster-aggregative nucleation mechanism occurred as temperature increased $\left(100{ }^{\circ} \mathrm{C}\right.$ as reported in figure S1), confirmed by the heterogeneous distribution of Ag NPs obtained after $35 \mathrm{~s}$ and $70 \mathrm{~s}$ of MW irradiation. It is also likely that an increase of reaction temperature gives rise to the desorption of the negatively charged ligand chains [27], promoting aggregation and polydispersity.

Furthermore, the uniformity of the PAA-Ag NPs synthesized under $17 \mathrm{~s}$ of MW irradiation was reflected in a higher stability for longer storage time, due to a reduction of Ostwald ripening and coalescence mechanisms [28]. The evolution of ultra-small particles in water suspension is still a common critical factor [29,30]. In fact, after a storage period as long as 18 months an evident alteration of the colloidal solution colour was observed for all the colloids synthesized. However, while at $35 \mathrm{~s}$ and $70 \mathrm{~s}$ a gradual transition to green colour was observed, the NPs produced in $17 \mathrm{~s}$ still presented a blue tone (optical images in Fig. 2). The TEM analysis confirmed that a drastic variation of particle size distribution was observed for $70 \mathrm{~s}$ and $35 \mathrm{~s}$ respectively, where particle size distribution shifted from a distribution centred in $2 \mathrm{~nm}$ with few NPs of around $12 \mathrm{~nm}$ to a wide distribution from 2 to $26 \mathrm{~nm}$ (Fig. 2b and d respectively). Instead, a higher uniformity was observed for the $17 \mathrm{~s}$ sample as clearly seen in Fig. 2f, where still $51 \%$ of the particles presented a diameter smaller than $3 \mathrm{~nm}$ and no particles bigger than $7 \mathrm{~nm}$ were observed after 18 months. The higher the homogeneity the lower the particles growth, confirming the theoretical model reported by Reiss [3] who theorized that smaller particles may grow faster in the presence of bigger particle. The results were also confirmed by UV-VIS spectroscopy. After synthesis (Fig. 2g) there was a unique long-wave absorption band at $\lambda>600 \mathrm{~nm}$ indicating the complexes of small particles with the carboxylate group of the polymer [27], without significant differences between the three samples. No variations were observed after 3 days (figure S2). On the other hand, after 18 months a secondary Ag NPs signal at a wavelength of $430 \mathrm{~nm}$ appeared exclusively for $70 \mathrm{~s}$ and $35 \mathrm{~s}$.

Considering the results reported above, we adopted the reference temperature of $74.6{ }^{\circ} \mathrm{C}$ with a conventional heating (CH) using an oil bath, to evaluate the different heating mechanisms. The evolution of the average synthesis temperature and its corresponding deviation, considering the measurements at the three different points of the vessel (see Fig. 1), under $\mathrm{CH}$ and MW is presented in Fig. 3. It shows different 

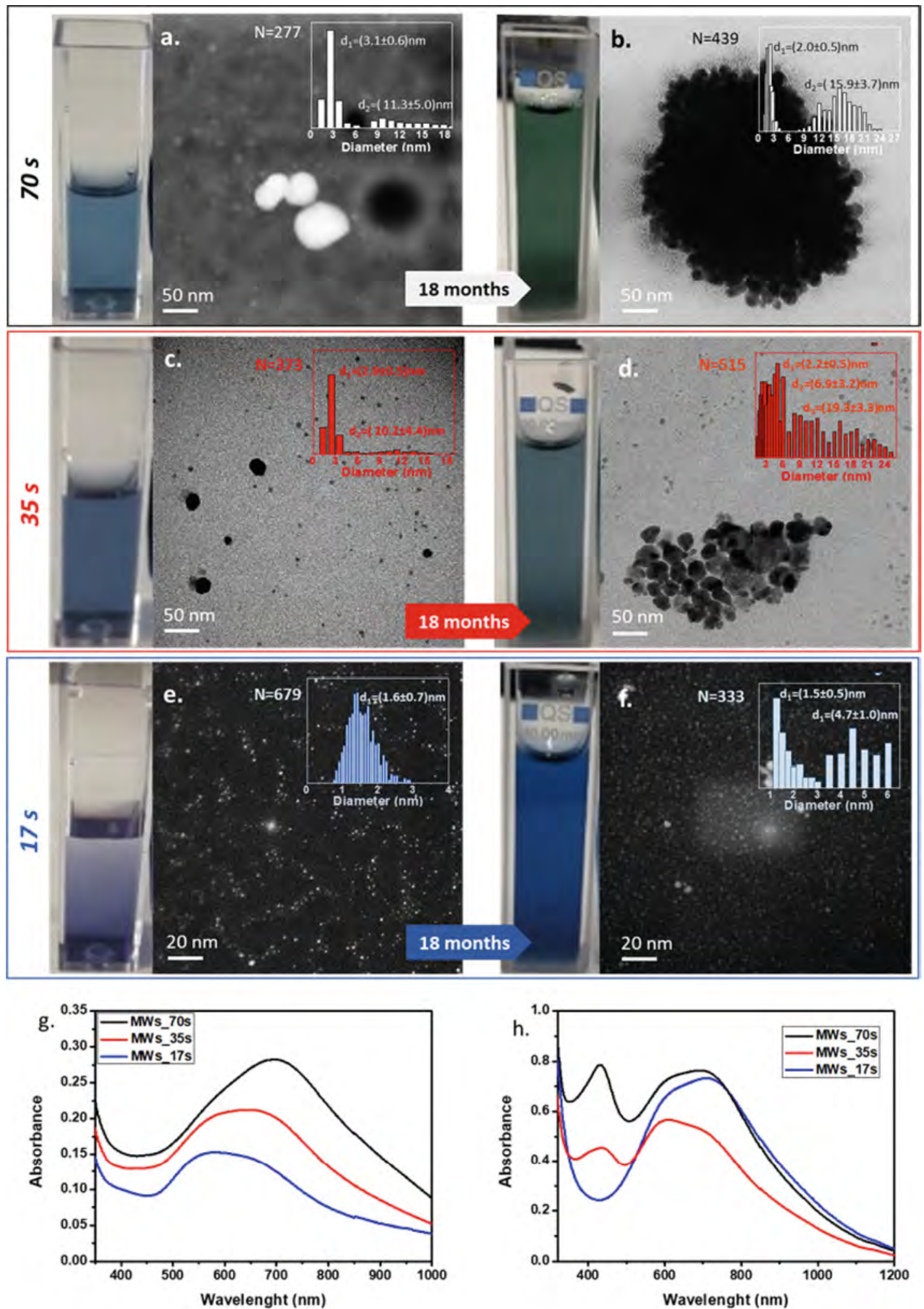

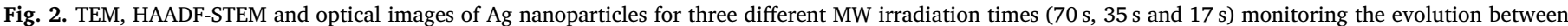

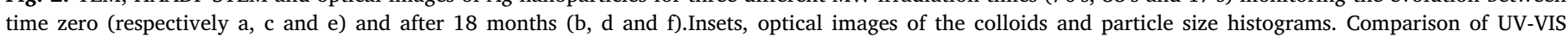
spectroscopy for three different MW irradiation times (70 s, $35 \mathrm{~s}$ and $17 \mathrm{~s}$ ) between time zero ( $\mathrm{g}$ ) and after $18 \mathrm{months}(\mathrm{h})$.

heating pattern and heating rate for the two heating systems to reach the same final set point. MW heating not only reached the final temperature 10 times faster, but a completely different heat transfer mechanism was observed. For $\mathrm{CH}$ (see Fig. 3b), it was possible to appreciate how the heating proceeded from the wall of the quartz reactor towards the centre with the hottest region close to the vessel wall. Instead, an opposite gradient was observed for MW heating, where the centre of the sample was rapidly heated and then the rest of the liquid underwent fast heating, with the high temperature wave moving towards the reactor wall. From LaMer theory [5], a control of the 


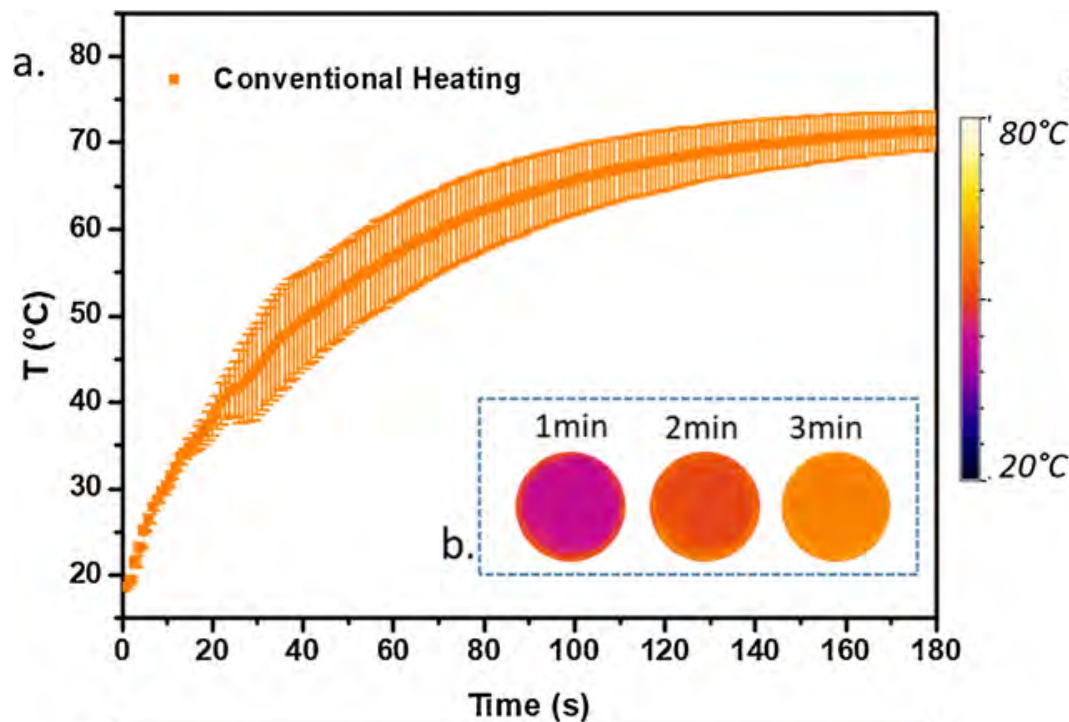

C.

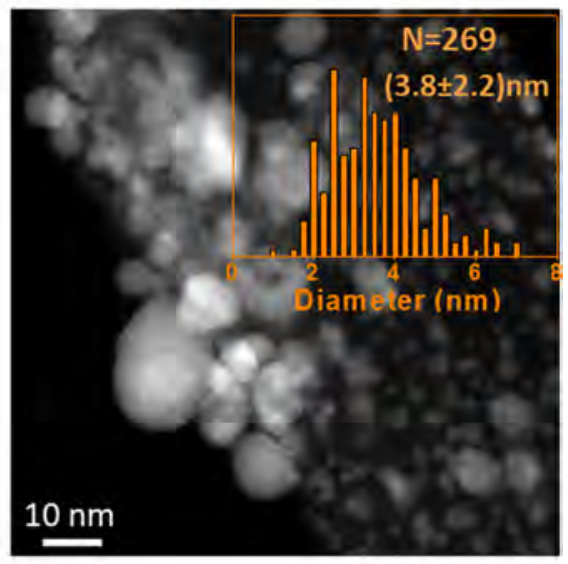

f.

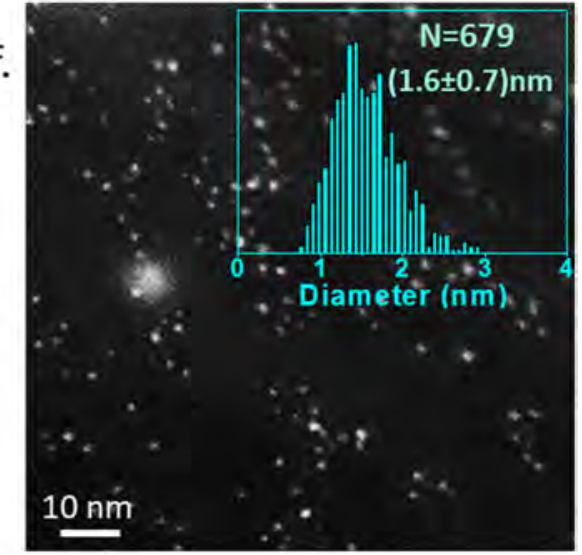

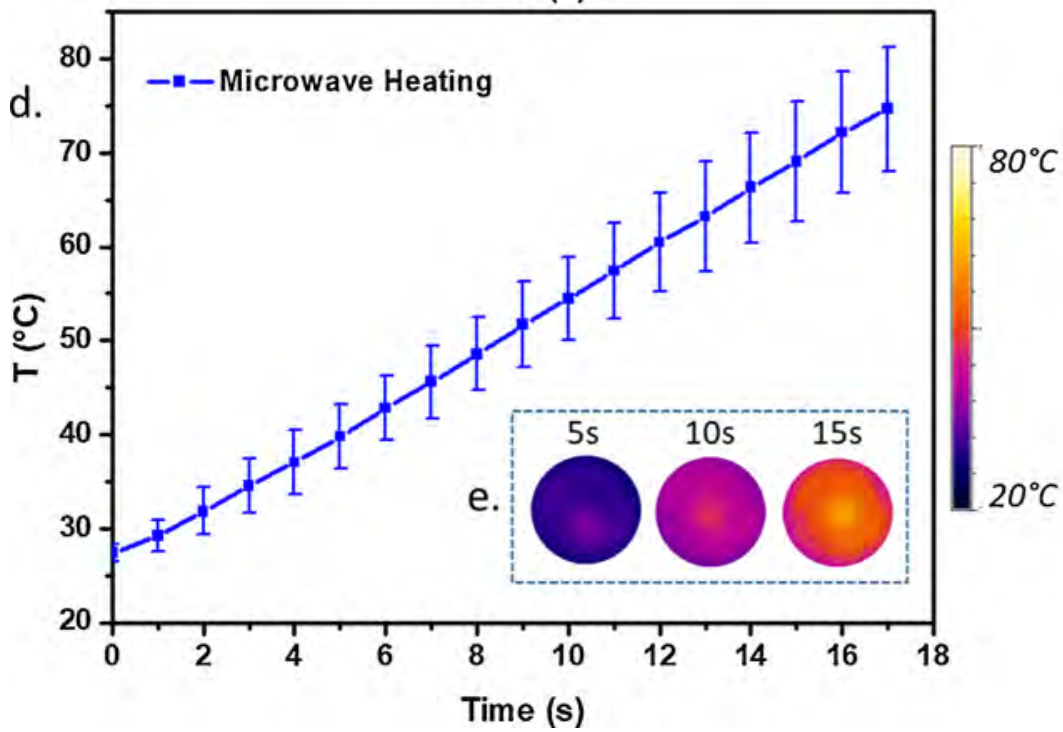

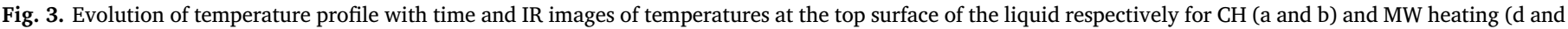
e). HAADF-STEM images and particle size histogram of Ag NPs for CH (c) and MW heating (f) respectively.

nucleation step, without the presence of successive nucleations, can be considered as essential to better control the homogeneity of the resulting NPs, and this gives a clear advantage to MW-heated systems, where the whole system is heated up to the required temperature in about $15 \mathrm{~s}$, while under conventional heating the centre of the vessel is still at $(58.7 \pm 5.7)^{\circ} \mathrm{C}$ after one minute of reaction. The fast nucleation under MW irradiation indeed results in a more homogeneous product, with a reduction of $70 \%$ of the NPs size deviation and a decrease of $58 \%$ of the NPs average diameter, as reported in Fig. $3 c$ and d respectively. It can be concluded that fast, volumetric heating is able to produce almost simultaneous nucleation in the whole reactor volume, while the slower conventional heating process gives rise to a heating wave that moves towards the centre of the vessel, producing new nucleation events as the heat front reaches inner regions. In the meantime, the nuclei formed at the outer regions continue to grow, consuming precursors and giving rise to a non-homogeneous growth in the vessel. These results are in agreement with the previous work of Liu et al. [31] confirming the thermal advantages of microwave heating. Indeed, the ability of MW heating to provide fast heating throughout the sample volume can be the key to control nanoparticle production in systems where fast kinetics are involved, such as in the production of noble metal nanoparticles where it is estimated that nucleation occurs in less than
$100 \mathrm{~ms}$ [32].

In order to confirm the influence of the heating rate, we gradually reduced the microwave power adopted for reaching a temperature of around $75{ }^{\circ} \mathrm{C}$ decreasing the heating rate from 2.8 to $0.2^{\circ} \mathrm{C} / \mathrm{s}$ as reported in Fig. 4 a), which evidently results also in an increase of synthesis time. A relevant variation of the size distribution was confirmed by UV-VIS spectroscopy in Fig. 4 b), where we observed a gradual increase of secondary signal of bigger silver nanoparticles at a wavelength of $425 \mathrm{~nm}$. The presence of bigger agglomerates was confirmed by TEM analysis in Fig. 4, where the slower the heating rate the higher the polydispersity. This result may confirm that the enhancement of the homogeneity of ultra-small nanoparticles reported by microwave heating is exclusively connected to a thermal effect, which is the fast heating rate that is difficult to achieve with other heating sources following conventional heating mechanisms.

\subsection{Supporting of Ultra-Small Ag nanoparticles on a mesoporous substrate}

Supporting metal NPs is essential to increase their stability and also to facilitate their application for heterogeneous catalysis. In this case, Ag-NPs produced under MW heating for $17 \mathrm{~s}$, were deposited on aminofunctionalized SBA-15 by wet impregnation. As reported in Figs. 5 a) 

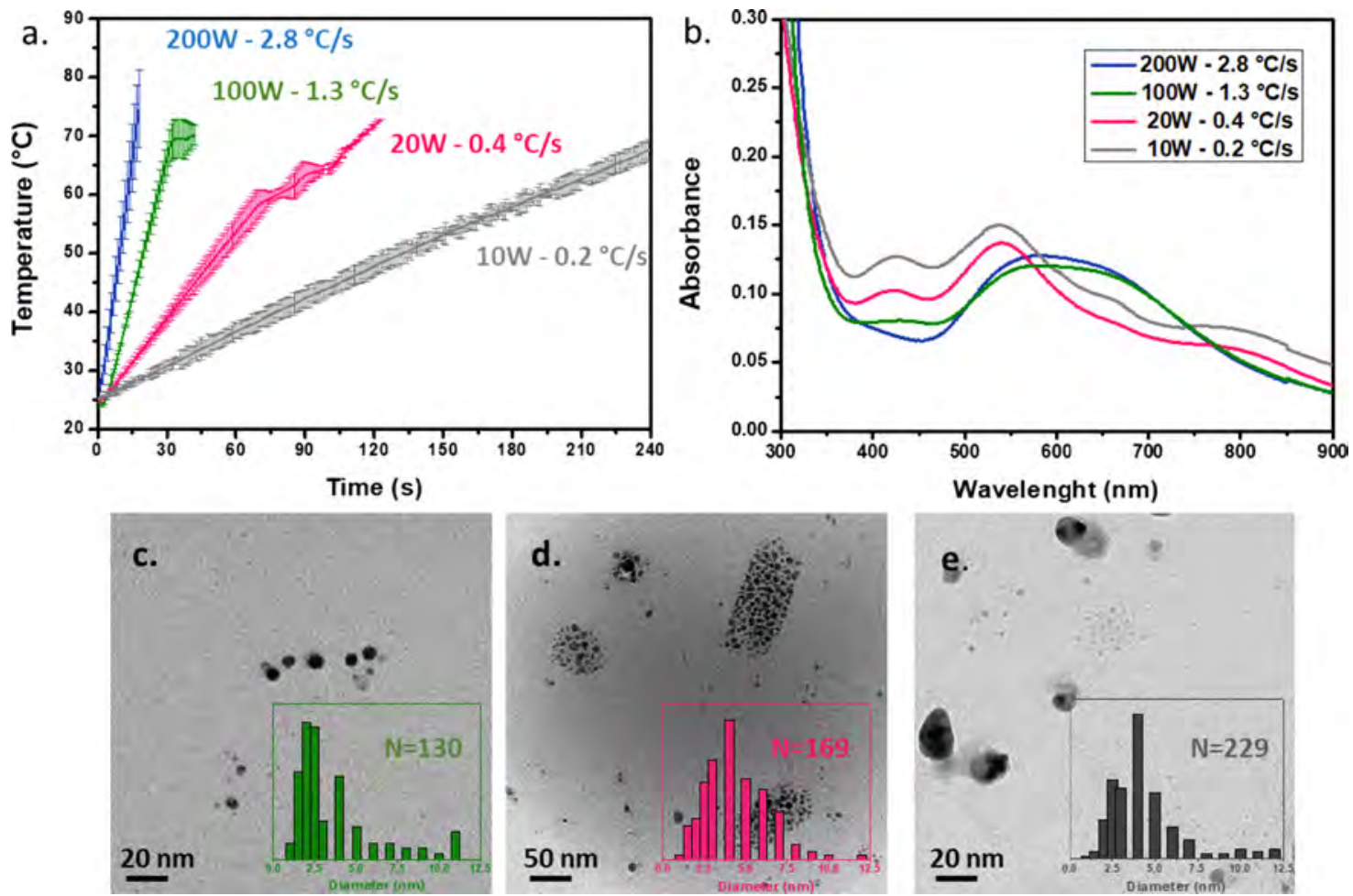

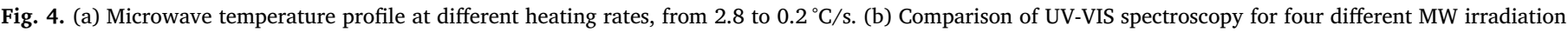
powers $(200 \mathrm{~W}, 100 \mathrm{~W}, 20 \mathrm{~W}$ and $10 \mathrm{~W}$ ). (c) TEM images at $100 \mathrm{~W}$, (d) TEM images at $20 \mathrm{~W}$ and (e) TEM images at $10 \mathrm{~W}$.

and b), a homogeneous arrangement of particles along the channels of the porous substrate was evidenced by HAADF-STEM analysis without relevant variation of their size distribution. The percentage of metal loading was quantified by MP-AES analysis as a total of $(0.98 \pm 0.05)$ $\%$ wt. Furthermore, no obstruction of the pores was evidenced by $\mathrm{N}_{2}$ adsorption analysis before and after the deposition of the NPs, as
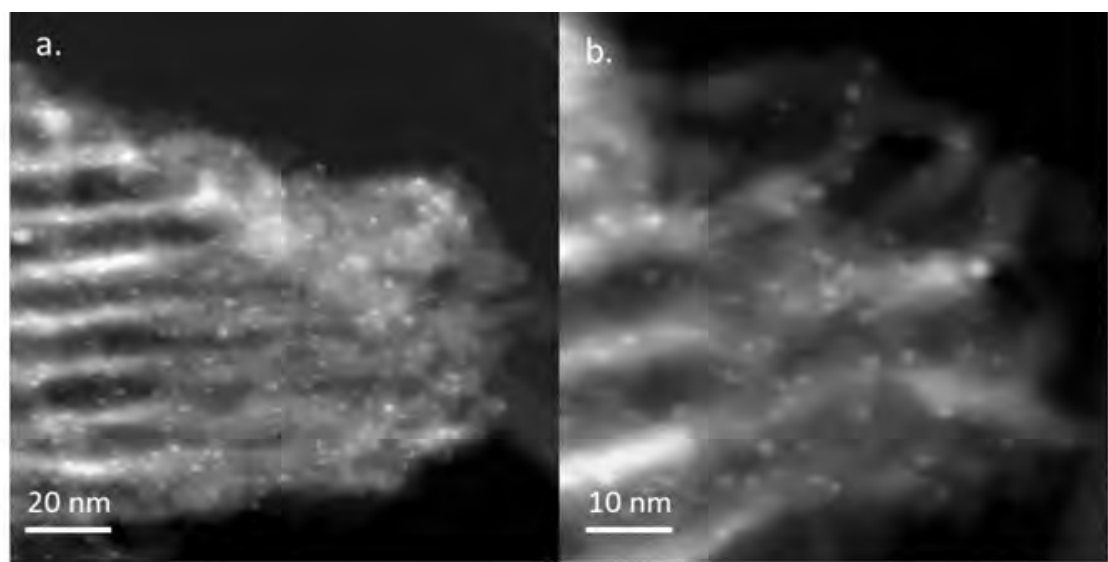

Fig. 5. A uniform distribution of PAAAg NPs over the channels of mesoporous SBA-15 substrate was evidenced by HAADF-STEM analysis ( $\mathrm{a}$ and $\mathrm{b}$ ). No relevant variations of pore diameter were observed after the grafting of AgNPs (c). Furthermore, XPS analysis confirmed the presence of both $\mathrm{Ag}(0)$ and $\operatorname{Ag}(\mathrm{I})$, complexed by the polycarboxylate group of PAA (d).
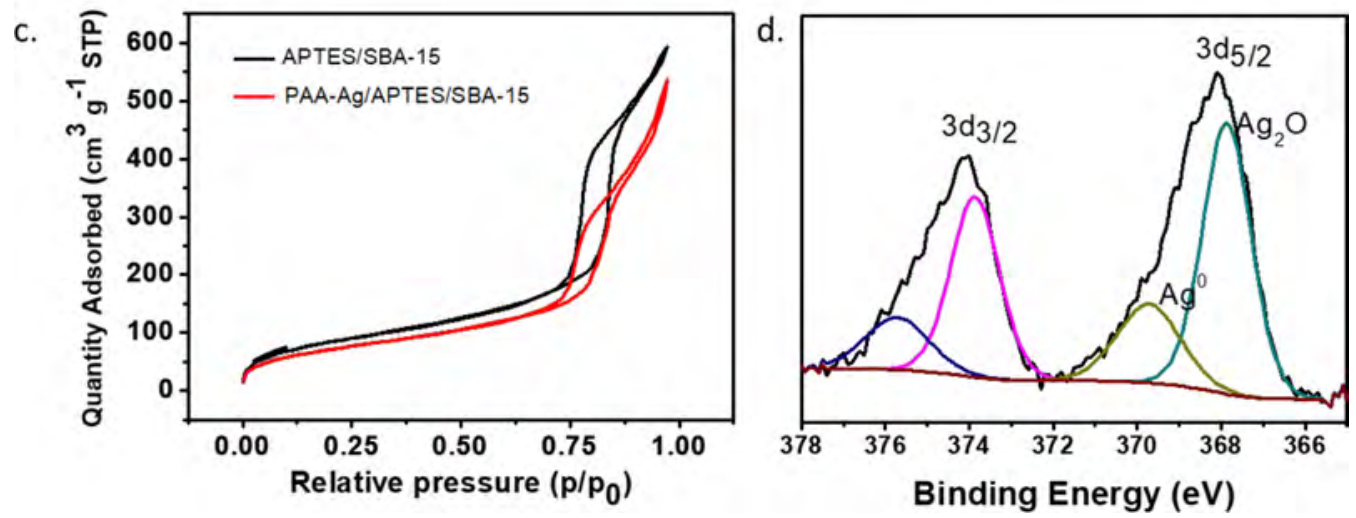

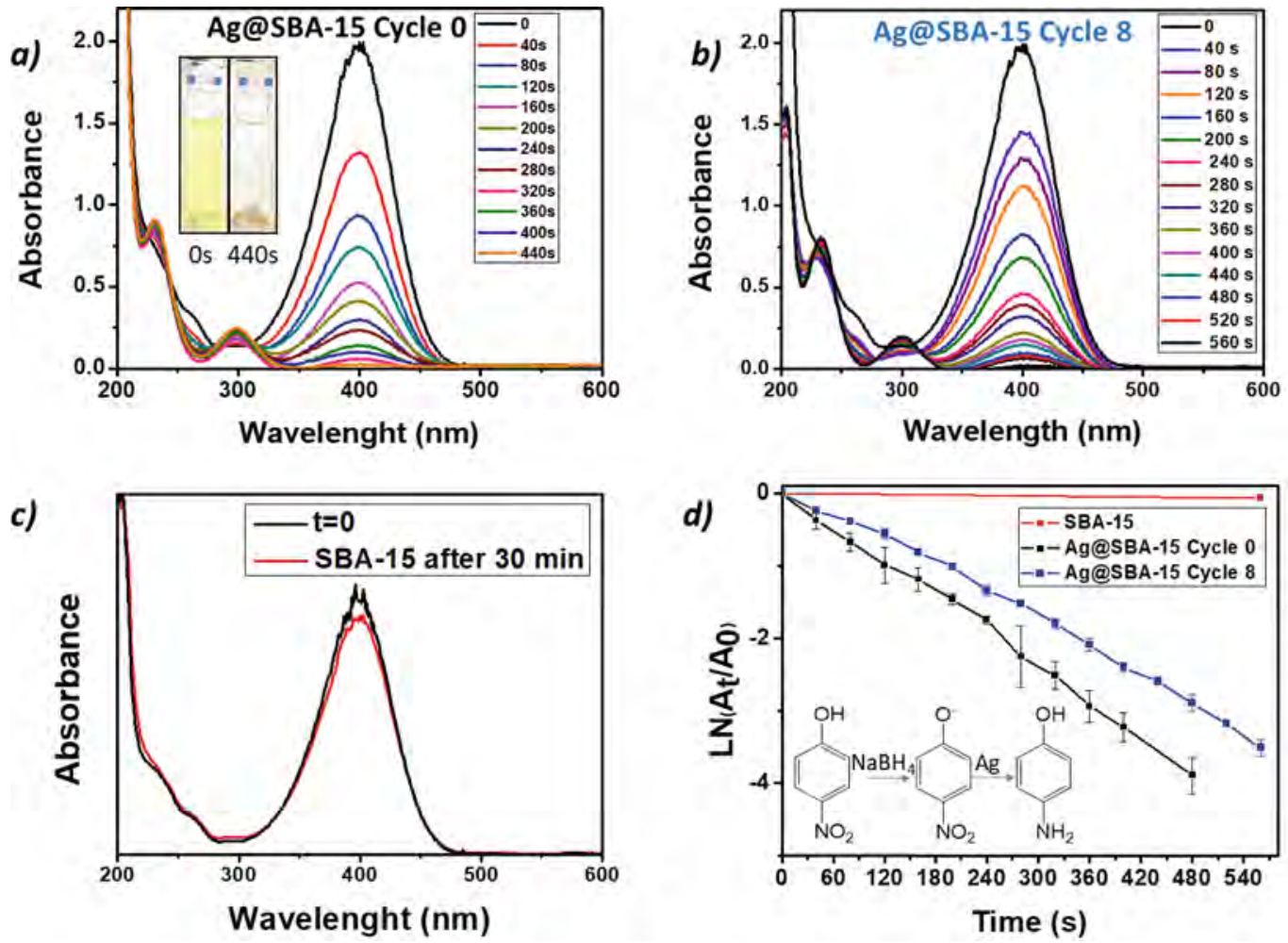

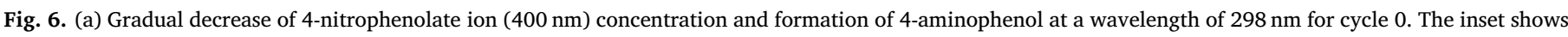

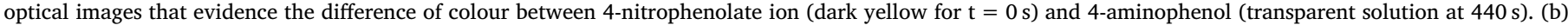

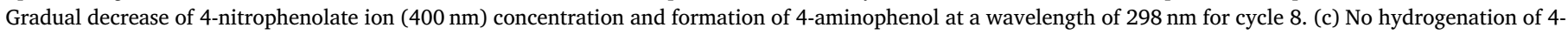

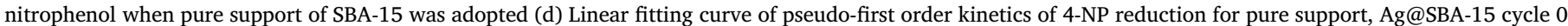
and Ag@SBA-15 cycle 8.

confirmed by the type IV isotherms in Fig. $5 \mathrm{c}$ ). The total pore volume in the sample containing the PAA-Ag NPs $\left(0.76 \mathrm{~cm}^{3} / \mathrm{g}\right)$ decreased only by $15 \%$ respect to the original SBA- 15 support (Table S1 and Figure S3). A uniform organization of the PAA-Ag NPs, together with an average pore size of $8.6 \mathrm{~nm}$ is highly desirable to ensure the access of a wide range of organic molecules to the active sites. $\mathrm{N}_{2}$ adsorption analysis details are presented in table S1 and Figure S3. The XPS analysis confirmed the presence of a core structure $(55 \%$ of $\mathrm{Ag}(0))$ surrounded by a high concentration of uncoordinated atoms $(45 \%$ of $\mathrm{Ag}(\mathrm{I})$ ), as reported in Fig. 5 d). The presence of a large proportion of atoms with a higher oxidation states around a noble metal core is not surprising, given the high surface to volume ratio of the NPs prepared in this case, and has been observed for other noble metals, e.g. Pt [33].

\subsection{Catalytic Activity}

Considering the adverse effects on human beings and aquatic life of the carcinogenic pollutant 4-Nitrophenol, the catalytic activity of AgNPs was tested for the reduction of 4-Nitrophenol in an excess of $\mathrm{NaBH}_{4}$. As reported in Fig. 6a), we firstly observed the formation of 4nitrophenolate ion (wavelength absorbance at $400 \mathrm{~nm}$, see the time $0 \mathrm{~s}$ curve in Fig. 6a and 6b). Only after the addition of the nanocatalyst the characteristic absorbance peak of 4-nitrophenolate gradually decreased while the peak of 4-aminophenol $(294 \mathrm{~nm})$ increased [34]. The reaction was concluded after $440 \mathrm{~s}$, as confirmed by the disappearance of the absorbance signal of sodium phenolate and the transparency of the solution, Fig. 5b. No reactions occurred in the absence of catalyst or with the pure mesoporous substrate SBA-15 (Fig. 6 c), confirming that Ag-NPs are responsible of the catalytic reaction. Langmuir- Hinshelwood kinetics were employed to quantify the catalytic performance [35]. The catalytic reduction reaction of 4-Nitrophenol to 4-Aminophenol has been well studied and considering that $\mathrm{NaBH}_{4}$ is in excess (C
$\mathrm{NaBH}_{4} / \mathrm{C}_{4-\text { nitrophenol }}=400$ ) it can be considered constant during the reaction process, and the reaction kinetics can be treated as pseudofirst-order with respect to 4-Nitrophenol. The apparent kinetic constant corresponded to the slope of the linear relation between the $\ln \left(A_{t} / A_{0}\right)$ and the reaction time. The linear correlation was excellent, with a fitting regression coefficient $r^{2}$ higher than 0.9935 for the three different batches, confirming the pseudo-first-order reaction kinetics (Figure S4). As confirmed by reusability test, the catalyst presented a good activity even after 8 cycles as reported in Fig. 6 b. We reported a reduction of $33 \%$ of kinetic constant if compared to fresh catalyst, with a kinetic constant of $(5.91 \pm 0.07) \mathrm{E}-03\left(1 \cdot \mathrm{mg}^{-1} \cdot \mathrm{s}^{-1}\right)$ (Fig. $\left.6 \mathrm{~d}\right)$. However, the lower catalytic activity observed after 8 cycles could be also attributed to gradual slight losses of catalyst in the recovery process with a centrifugation step between the different cycles.

Table 1 compares the normalized kinetic constant of several works reported in literature where Ag NPs were used as a catalyst for 4-Nitrophenol abatement. The low diameter $(1.6 \pm 0.7) \mathrm{nm}$ and the uniform distribution of the Ag NPs on the surfaces of the mesoporous substrate strongly impacted on the resulted catalytic activity, which was around two times higher than the best Ag-based catalyst listed in Table 1 [36]. This in turn allowed to operate with a considerable reduction of metal loading leading to enviromental and economical advantages [37].

\section{Conclusions}

The fast heating rate and volumetric character of MW heating were essential for the synthesis of ultra-small Ag NPs with a uniform size distribution of $(1.6 \pm 0.7) \mathrm{nm}$, obtained after $17 \mathrm{~s}$ synthesis time. An increase of the synthesis temperature and time may lead to a lower homogeneity of the product which is likely due to a higher number of clusters coalescing that would lead to a broader particle size 
Table 1

A summary of Normalized Kinetic Constant for the reduction of 4-NP catalysed by Ag nanoparticles.

\begin{tabular}{|c|c|c|c|c|}
\hline Catalyst & Diameter (nm) & $\begin{array}{l}{[\mathrm{Ag}](\mathrm{mg}} \\
\left.\mathrm{l}^{-1}\right)\end{array}$ & $\mathrm{K}\left(1 \mathrm{mg}^{-1} \mathrm{~s}^{-1}\right)$ & Ref. \\
\hline $\mathrm{Ag} / \mathrm{CaCO}_{3}$ & $20-30$ & 1250 & $2.54 \mathrm{E}-06$ & [38] \\
\hline$A g N P s$ & $10-35$ & 616 & $4.30 \mathrm{E}-06$ & [34] \\
\hline$A g N P / C$ & $10.0 \pm 2.0$ & 184 & $9.18 \mathrm{E}-06$ & [39] \\
\hline Chitosan-Ag NPs & $\sim 3$ & 273 & $9.82 \mathrm{E}-06$ & [40] \\
\hline Ag NPs-CP25 & $30-40$ & 975 & $1.60 \mathrm{E}-05$ & [41] \\
\hline Ag NPs & $540-640$ & 500 & $2.00 \mathrm{E}-05$ & [42] \\
\hline PI/Ag NPs & $5-20$ & 344 & $2.75 \mathrm{E}-05$ & [43] \\
\hline$A g / r G O N P s^{*}$ & $8.6 \pm 3.5$ & 250 & 4.06E-05 & [44] \\
\hline EPS-Ag & $\sim 5$ & 27 & 4.73E-05 & [45] \\
\hline $\mathrm{TA} @ \mathrm{Fe}_{3} \mathrm{O}_{4}-\mathrm{AgNPs}$ & $\sim 10$ & 200 & $5.35 \mathrm{E}-05$ & [46] \\
\hline$A g-O M S-C$ & - & 390 & 7.77E-05 & [47] \\
\hline Ag/SiO2/PNIPAM & $15-20$ & 46 & 8.99E-05 & [48] \\
\hline$A g @ C$ & $6-8$ & 78 & $1.81 \mathrm{E}-04$ & [35] \\
\hline$S P A G$ & $\sim 50$ & 8.5 & $9.45 \mathrm{E}-04$ & [49] \\
\hline Ag@mTiO2@CF & $\sim 10$ & 13 & $1.44 \mathrm{E}-03$ & [50] \\
\hline$A g / S B A-15$ & $\sim 7$ & 2.3 & $1.56 \mathrm{E}-03$ & [51] \\
\hline$A g N P-P G-25 K$ & $85.0 \pm 2.0$ & 3.5 & $1.57 \mathrm{E}-03$ & [52] \\
\hline AgNPs@PGMA-SH & $17.0 \pm 3.2$ & 0.9 & $4.38 \mathrm{E}-03$ & [36] \\
\hline Ag-NPs/SBA-15 & $1.6 \pm 0.7$ & 1 & $(7.90 \pm 0.58) \mathrm{E}-03$ & This work \\
\hline
\end{tabular}

distribution, as observed for longer heating times of $35 \mathrm{~s}$ and $70 \mathrm{~s}$. Furthermore, the homogeneity of the suspension formed with small nanoparticle clusters assured a longer storage life-time of the product, without the observation of the characteristic plasmon peak of silver nanoparticles at $400 \mathrm{~nm}$, after 18 months storage. MW heating resulted in a 10 times faster heating rate, compared to $\mathrm{CH}$ in an oil bath. MW heating resulted in higher homogeneity of NPs with a 70\% reduction of the particle size deviation and also a reduction of $58 \%$ of the average diameter. The Ag NPs were deposited on the porous surface of SBA-15 (1\%wt.) by wet impregnation, giving rise to a homogeneous particle distribution both inside and outside the pores, while preserving accessibility of the reactants to the mesoporous structure. The catalytic activity of the Ag-NPs/SBA-15 was tested in the reduction of 4Nitrophenol. A high value of the kinetic constant for the pseudo-first order reaction was observed of $(7.90 \pm 0.58) \mathrm{E}-03\left(1 \cdot \mathrm{mg}^{-1} \cdot \mathrm{s}^{-1}\right)$ which is above the values previously reported for this reaction.

\section{Declaration of Competing Interest}

The authors declare that they have no known competing financial interests or personal relationships that could have appeared to influence the work reported in this paper.

\section{CRediT authorship contribution statement}

Roberta Manno: Investigation, Data curation, Writing - original draft. Victor Sebastian: Conceptualization, Supervision, Writing - review \& editing. Silvia Irusta: Data curation. Reyes Mallada: Writing review \& editing, Supervision. Jesús Santamaria: Writing - review \& editing, Supervision, Funding acquisition.

\section{Acknowledgements}

This project has received funding from the European Union's Horizon 2020 research and innovation programme under the Marie Skłodowska-Curie grant agreement No 721290. This publication reflects only the author's view, exempting the Community from any liability. Project website: http://cosmic-etn.eu/. The microscopy works have been conducted in the Laboratorio de Microscopias Avanzadas at Instituto de Nanociencia de Aragón-Universidad de Zaragoza. Authors acknowledge the LMA-INA for offering access to their instruments and expertise.

\section{Appendix A. Supplementary data}

Supplementary material related to this article can be found, in the online version, at doi:https://doi.org/10.1016/j.cattod.2020.04.018.

\section{References}

[1] R. Jin, C. Zeng, M. Zhou, Y. Chen, Atomically Precise Colloidal Metal Nanoclusters and Nanoparticles: Fundamentals and Opportunities, Chem. Rev. 116 (2016) 10346-10413, https://doi.org/10.1021/acs.chemrev.5b00703.

[2] B. Calderón-Jiménez, M.E. Johnson, A.R. Montoro Bustos, K.E. Murphy, M.R. Winchester, J.R.V. Baudrit, Silver nanoparticles: Technological advances, societal impacts, and metrological challenges, Front. Chem. 5 (2017) 1-26, https:// doi.org/10.3389/fchem.2017.00006.

[3] J. Polte, Fundamental growth principles of colloidal metal nanoparticles - a new perspective, CrystEngComm. 17 (2015) 6809-6830, https://doi.org/10.1039/ c5ce01014d.

[4] R. Becker, W. Döring, Kinetische Behandlung der Keimbildung in übersättigten Dämpfen, Ann. Phys. 416 (1935) 719-752, https://doi.org/10.1002/andp. 19354160806.

[5] M. Keating, Y. Chen, I.A. Larmour, K. Faulds, D. Graham, Growth and surface-enhanced Raman scattering of Ag nanoparticle assembly in agarose gel, Meas. Sci. Technol. 23 (2012) 084006-0840015, https://doi.org/10.1088/0957-0233/23/8/ 084006.

[6] L. Liu, A. Corma, Metal Catalysts for Heterogeneous Catalysis: From Single Atoms to Nanoclusters and Nanoparticles, Chem. Rev. 118 (2018) 4981-5079, https://doi. org/10.1021/acs.chemrev.7b00776.

[7] N.T.K. Thanh, N. Maclean, S. Mahiddine, Mechanisms of nucleation and growth of nanoparticles in solution, Chem. Rev. 114 (2014) 7610-7630, https://doi.org/10. $1021 / \mathrm{cr} 400544 \mathrm{~s}$.

[8] C. Burda, X. Chen, R. Narayanan, M.A. El-Sayed, Chemistry and properties of nanocrystals of different shapes, (2005), https://doi.org/10.1021/cr030063a.

[9] J. Belloni, M. Mostafavi, Radiation chemistry of nanocolloids and clusters, Stud. Phys. Theor. Chem. 87 (2001) 411-452, https://doi.org/10.1016/S0167-6881(01) 80018-5.

[10] M. Mostafavi, G.R. Rey, L. François, J. Belloni, Transient and Stable Silver Clusters Induced by Radiolysis in Methanol, J. Phys. Chem. A. 106 (2002) 10184-10194 https://doi.org/10.1021/jp014257a.

[11] S. Remita, J.M. Orts, J.M. Feliu, M. Mostafavi, M.O. Delcourt, STM identification of silver oligomer clusters prepared by radiolysis in aqueous solution, Chem. Phys. Lett. 218 (1994) 115-121, https://doi.org/10.1016/0009-2614(93)E1451-L.

[12] S. Liu, F. Lu, J.-J. Zhu, Highly fluorescent Ag nanoclusters: microwave-assisted green synthesis and Cr3 + sensing, Chem. Commun. 47 (2011) 2661-2663, https:// doi.org/10.1039/c0cc04276e.

[13] W. Tu, H. Liu, Continuous synthesis of colloidal metal nanoclusters by microwave irradiation, Chem. Mater. 12 (2000) 564-567, https://doi.org/10.1021/ cm9906371.

[14] I. Díez, M. Pusa, S. Kulmala, H. Jiang, A. Walther, A.S. Goldmann, A.H.E. Müller, O. Ikkala, R.H.A. Ras, Color tunability and electrochemiluminescence of silver nanoclusters, Angew. Chemie - Int. Ed. 48 (2009) 2122-2125, https://doi.org/10. 1002/anie.200806210.

[15] H. Xu, K.S. Suslick, Sonochemical Synthesis of Highly Fluorescent Ag Nanoclusters, ACS Nano. 4 (2010) 3209-3214, https://doi.org/10.1021/nn100987k.

[16] Y. Yang, C. Ochoa-Hernández, V.A. De La Peña O’Shea, J.M. Coronado, D.P. Serrano, Ni2P/SBA-15 as a hydrodeoxygenation catalyst with enhanced selectivity for the conversion of methyl oleate into n-octadecane, ACS Catal. 2 (2012) 592-598, https://doi.org/10.1021/cs200659r.

[17] Y.T. Lai, T.C. Chen, Y.K. Lan, B.S. Chen, J.H. You, C.M. Yang, N.C. Lai, J.H. Wu, C.S. Chen, Pt/SBA-15 as a highly efficient catalyst for catalytic toluene oxidation, ACS Catal. 4 (2014) 3824-3836, https://doi.org/10.1021/cs500733j.

[18] S. Zhang, S. Muratsugu, N. Ishiguro, M. Tada, Ceria-Doped Ni/SBA-16 Catalysts for Dry Reforming of Methane, ACS Catal. 3 (2013), https://doi.org/10.1021/ cs400159w 1885-1864.

[19] D. Pangkita, B. Debajyoti, S. Pingal, D. Ramesh C, B. Pankaj, Catalytic Reduction of Water Contaminant '4-Nitrophenol' over Manganese Oxide Supported Ni Nanoparticles, Trends Asian Water Environ. Sci. Technol. (2017) 250, https://doi. org/10.1007/978-3-319-39259-2.

[20] A. Kumar, M. Belwal, R.R. Maurya, V. Mohan, V. Vishwanathan, Heterogeneous catalytic reduction of anthropogenic pollutant, 4-nitrophenol by Au/AC nanocatalysts, Mater. Sci. Energy Technol. 2 (2019) 526-531, https://doi.org/10.1016/j. mset.2019.05.007.

[21] National Research Council, Drinking Water and Health 4 (1982), https://doi.org/ $10.17226 / 325$.

[22] L. Uson, J.L. Hueso, V. Sebastian, R. Arenal, I. Florea, S. Irusta, M. Arruebo, J. Santamaria, In-situ preparation of ultra-small Pt nanoparticles within rod-shaped mesoporous silica particles: 3-D tomography and catalytic oxidation of n-hexane, Catal. Commun. 100 (2017) 93-97, https://doi.org/10.1016/j.catcom.2017.06. 022 .

[23] G.S.J. Sturm, M.D. Verweij, T. Van Gerven, A.I. Stankiewicz, G.D. Stefanidis, On the effect of resonant microwave fields on temperature distribution in time and space, Int. J. Heat Mass Transf. 55 (2012) 3800-3811, https://doi.org/10.1016/j. ijheatmasstransfer.2012.02.065.

[24] M. Takesue, T. Tomura, M. Yamada, K. Hata, S. Kuwamoto, T. Yonezawa, Size of elementary clusters and process period in silver nanoparticle formation, J. Am. 
Chem. Soc. 133 (2011) 14164-14167, https://doi.org/10.1021/ja202815y.

[25] A.N. Naik, S. Patra, D. Sen, A. Goswami, Evaluating the mechanism of nucleation and growth of silver nanoparticles in a polymer membrane under continuous precursor supply: Tuning of multiple to single nucleation pathway, Phys. Chem. Chem. Phys. 21 (2019) 4193-4199, https://doi.org/10.1039/c8cp06202a.

[26] A. Dutta, A. Paul, A. Chattopadhyay, The effect of temperature on the aggregation kinetics of partially bare gold nanoparticles, RSC Adv. 6 (2016) 82138-82149, https://doi.org/10.1039/c6ra17561a.

[27] B.M. Sergeev, L.I. Lopatina, A.N. Prusov, G.B. Sergeev, Formation of silver clusters by borohydride reduction of $\mathrm{AgNO} 3$ in polyacrylate aqueous solutions, Colloid J. 67 (2005) 72-78, https://doi.org/10.1007/s10595-005-0008-5.

[28] S. Hu, W.X. Li, Influence of Particle Size Distribution on Lifetime and Thermal Stability of Ostwald Ripening of Supported Particles, ChemCatChem. 10 (2018) 2900-2907, https://doi.org/10.1002/cctc.201800331.

[29] T.M.D. Dang, T.T.T. Le, E. Fribourg-Blanc, M.C. Dang, The influence of solvents and surfactants on the preparation of copper nanoparticles by a chemicalreduction method, Adv. Nat. Sci. Nanosci. Nanotechnol. 2 (2011) 25004-25011, https://doi. org/10.1088/2043-6262/2/2/025004.

[30] B.G. Nikolovski, J.D. Ilić, M.N. Sovilj, How to formulate a stable and monodisperse water-in-oil nanoemulsion containing pumpkin seed oil: The use of multiobjective optimization, Brazilian J. Chem. Eng. 33 (2016) 919-931, https://doi.org/10. 1590/0104-6632.20160334s20140140.

[31] W. Tu, H. Liu, Rapid synthesis of nanoscale colloidal metal clusters by microwave irradiation, J. Mater. Chem. 10 (2000) 2207-2211, https://doi.org/10.1039/ b002232m.

[32] J. Polte, X. Tuaev, M. Wuithschick, A. Fischer, A.F. Thuenemann, K. Rademann, R. Kraehnert, F. Emmerling, Formation mechanism of colloidal silver nanoparticles: Analogies and differences to the growth of gold nanoparticles, ACS Nano. 6 (2012) 5791-5802, https://doi.org/10.1021/nn301724z.

[33] Y. Li, J.H. Liu, C.A. Witham, W. Huang, M.A. Marcus, S.C. Fakra, P. Alayoglu, Z. Zhu, C.M. Thompson, A. Arjun, K. Lee, E. Gross, F.D. Toste, G.A. Somorjai, A PtCluster-Based Heterogeneous Catalyst for Homogeneous Catalytic Reactions : X-ray Absorption Spectroscopy and Reaction Kinetic Studies of Their Activity and Stability against Leaching, J. Am. Chem. Soc. 133 (2011) 13527-13533, https:// doi.org/10.1021/ja204191t.

[34] K. Muthu, S. Priya, Green synthesis, characterization and catalytic activity of silver nanoparticles using Cassia auriculata flower extract separated fraction, Spectrochim. Acta - Part A Mol. Biomol. Spectrosc. 179 (2017) 66-72, https://doi. org/10.1016/j.saa.2017.02.024.

[35] L.R. Shultz, L. Hu, K. Preradovic, M.J. Beazley, X. Feng, T. Jurca, A Broader-scope Analysis of the Catalytic Reduction of Nitrophenols and Azo Dyes with Noble Metal Nanoparticles, ChemCatChem. 11 (2019) 2590-2595, https://doi.org/10.1002/ cctc. 201900260 .

[36] W. Zhang, Y. Sun, L. Zhang, In Situ Synthesis of Monodisperse Silver Nanoparticles on Sulfhydryl-Functionalized Poly(glycidyl methacrylate) Microspheres for Catalytic Reduction of 4-Nitrophenol, Ind. Eng. Chem. Res. 54 (2015) 6480-6488, https://doi.org/10.1021/acs.iecr.5b01010.

[37] M.L. Crawley, B.M. Trost, Applications of Transition Metal Catalysis in Drug Discovery and Development: An Industrial Perspective, Wiley Online Library, 2012, https://doi.org/10.1002/9781118309872.

[38] S. Dash, S. Das, M.I. Khan, S. Sinha, B. Das, R. Jayabalan, P.K. Parhi, S.K. Tripathy, Sonochemically synthesized $\mathrm{Ag} / \mathrm{CaCO} 3$ nanocomposites: A highly efficient reusable catalyst for reduction of 4-nitrophenol, Mater. Chem. Phys. 220 (2018) 409-416, https://doi.org/10.1016/j.matchemphys.2018.09.019.

[39] S. Tang, S. Vongehr, X. Meng, Carbon spheres with controllable silver nanoparticle doping, J. Phys. Chem. C. 114 (2010) 977-982, https://doi.org/10.1021/ jp9102492.

[40] A. Murugadoss, A. Chattopadhyay, A "green" chitosan-silver nanoparticle composite as a heterogeneous as well as micro-heterogeneous catalyst, Nanotechnology. 19 (2008), https://doi.org/10.1088/0957-4484/19/01/015603.

[41] A. Dolatkhah, P. Jani, L.D. Wilson, Redox-Responsive Polymer Template as an Advanced Multifunctional Catalyst Support for Silver Nanoparticles, Langmuir. 34 (2018) 10560-10568, https://doi.org/10.1021/acs.langmuir.8b02336.

[42] S. Zhao, L. Duan, C. Xiao, L. Li, F. Liao, Single Metal of Silver Nanoparticles in the Microemulsion for Recyclable Catalysis of 4-Nitrophenol Reduction, J. Adv. Nanomater. 2 (2017) 31-40, https://doi.org/10.22606/jan.2017.21004.

[43] J. Li, Y. Wang, M. Wang, L. Wang, H. Li, A highly robust and reusable polyimidesupported nanosilver catalyst for the reduction of 4-nitrophenol, J. Mater. Res. 30 (2015) 2713-2721, https://doi.org/10.1557/jmr.2015.258.

[44] K.C. Hsu, D.H. Chen, Green synthesis and synergistic catalytic effect ofAg/reduced graphene oxide nanocomposite, Nanoscale Res. Lett. 9 (2014) 1-10, https://doi. org $/ 10.1186 / 1556-276 X-9-484$.

[45] Z. Zheng, Q. Huang, H. Guan, S. Liu, In situ synthesis of silver nanoparticles dispersed or wrapped by a Cordyceps sinensis exopolysaccharide in water and their catalytic activity, RSC Adv. 5 (2015) 69790-69799, https://doi.org/10.1039/ c5ra09452f.

[46] A. Sangili, M. Annalakshmi, S.M. Chen, P. Balasubramanian, M. Sundrarajan, Synthesis of silver nanoparticles decorated on core-shell structured tannic acidcoated iron oxide nanospheres for excellent electrochemical detection and efficient catalytic reduction of hazardous 4-nitrophenol, Compos. Part B Eng. 162 (2019) 33-42, https://doi.org/10.1016/j.compositesb.2018.10.084.

[47] H.T. Fan, X.G. Liu, X.J. Xing, B. Li, K. Wang, S.T. Chen, Z. Wu, D.F. Qiu, Ordered mesoporous silica cubic particles decorated with silver nanoparticles: A highly active and recyclable heterogeneous catalyst for the reduction of 4-nitrophenol, Dalt. Trans. 48 (2019) 2692-2700, https://doi.org/10.1039/c8dt04663h.

[48] J. Xu, T. Zhou, L. Jia, X. Shen, X. Li, H. Li, Z. Xu, J. Cao, Generation and thermally adjustable catalysis of silver nanoparticle immobilized temperature-sensitive nanocomposite, J. Nanoparticle Res. 19 (2017), https://doi.org/10.1007/s11051-017 3769-y.

[49] R.K. Narayanan, S.J. Devaki, Brawny silver-hydrogel based nanocatalyst for reduction of nitrophenols: Studies on kinetics and mechanism, Ind. Eng. Chem. Res. 54 (2015) 1197-1203, https://doi.org/10.1021/ie5038352.

[50] B.K. Ghosh, D. Moitra, M. Chandel, H. Lulla, N.N. Ghosh, Ag nanoparticle immobilized mesoporous TiO2-cobalt ferrite nanocatalyst: A highly active, versatile, magnetically separable and reusable catalyst, Mater. Res. Bull. 94 (2017) 361-370 https://doi.org/10.1016/j.materresbull.2017.06.015.

[51] B. Naik, S. Hazra, V.S. Prasad, N.N. Ghosh, Synthesis of Ag nanoparticles within the pores of SBA-15: An efficient catalyst for reduction of 4-nitrophenol, Catal. Commun. 12 (2011) 1104-1108, https://doi.org/10.1016/j.catcom.2011.03.028.

[52] B. Baruah, G.J. Gabriel, M.J. Akbashev, M.E. Booher, Facile synthesis of silver nanoparticles stabilized by cationic polynorbornenes and their catalytic activity in 4 nitrophenol reduction, Langmuir. 29 (2013) 4225-4234, https://doi.org/10.1021/ la305068p. 